\title{
Dimensionamento de um Sistema Robotizado para a Soldagem pelo Processo Friction Stir Welding
}

\author{
Bruno Silva Cota $^{1}$, Alexandre Queiroz Bracarense ${ }^{1}$, Fagner Guilherme Ferreira Coelho ${ }^{2}$ \\ 1 Universidade Federal de Minas Gerais - UFMG, Programa de Pós-Graduação em Engenharia Mecânica, Belo Horizonte, MG, Brasil. \\ 2 Comau do Brasil, Betim, MG, Brasil.
}

Recebido: 14 Nov., 2017

Aceito: 24 Nov., 2017

E-mail: brunocota@ufmg.br(BSC)
Este é um artigo publicado em acesso aberto (Open Access) sob a licença Creative Commons Attribution Non-Commercial, que permite uso, distribuição e reprodução em qualquer meio, sem restriçōes desde que sem fins comerciais e que 0 trabalho original seja corretamente citado.
Resumo: A primeira aplicação de um robô de soldagem pelo processo FSW (Friction Stir Welding) foi apresentada ao público em Gotemburgo, Suécia, no ano 2000, durante o Friction Stir Welding Symposium por dois diferentes grupos de pesquisa. Um grupo utilizava um robô antropomórfico modelo ABB IRB-6400 combinado a um motor elétrico de 220W e o outro um robô Neos de hastes paralelas. Apesar do processo FSW estar sendo objeto de estudo em vários centros de pesquisa no mundo, poucos estudam sua aplicação em robôs industriais, em que se destacam os institutos e as empresas: HGZ (Helmholtz-Zentrum Geesthacht), IWB e KUKA (em conjunto com o Grupo Airbus), na Alemanha; a ESAB, na Suécia; Institut Maupertuis e Institut de Soudure, na França; Cewac, na Bélgica; Friction Stir Link, nos Estados Unidos. Este trabalho tem como objetivo apresentar o estado da arte no que diz respeito à utilização do processo de soldagem FSW robotizado, além de evidenciar o processo realizado para o dimensionamento de um sistema completo para a soldagem robotizada utilizando um robô antropomórfico COMAU SMART NJ500, com 6 graus de liberdade e capacidade de 500 kg.

Palavras-chave: Friction stir welding; Soldagem; Robotização; Fricção; Industrial.

\section{Sizing of a Robot System for Joining by Friction Stir Welding Process}

Abstract: The first application of a FSW (Friction Stir Welding) robot was presented to the public in Gothenburg, Sweden, in 2000, during the Friction Stir Welding Symposium by two different research groups. One group used an ABB IRB-6400 anthropomorphic robot combined with a $220 \mathrm{~W}$ electric motor and the other a Neos robot with parallel rods. Although FSW is worldwide covered by many research groups, only few have been focusing on the implementation of the process on industrial robots. Some of the research groups and companies working on robotic FSW are HZG, Riftec, IWB and KUKA (jointly with Airbus Group) in Germany, ESAB in Sweden, Institut Maupertuis and Institut de soudure in France, Cewac in Belgium and Friction Stir Link in the USA. The objective of this article is to present the state of the art with regard to the use of the FSW welding process, besides evidencing the process carried out for the design of a complete system for robotized welding using a COMAU SMART NJ500 anthropomorphic robot with 6 degrees of freedom and capacity of $500 \mathrm{~kg}$.

Key-words: Friction stir welding; Welding; Robotization; Friction; Industrial.

\section{Introdução}

A soldagem é o mais importante método para a união permanente de metais, sendo utilizado desde os tempos remotos, como demonstram artefatos de ouro brasados na Pérsia e expostos no museu do Louvre, em Paris, que datam de 4000 a.C [1]. Verifica-se que as técnicas de soldagem evoluíram radicalmente desde as primeiras experiências de Sir Humphrey Davy com o arco elétrico no início do século XIX, e após a primeira patente obtida na Inglaterra por Nikolas Bernados e Stanislav Olszewsky em 1885, em que foi criado um dispositivo baseado no princípio do arco elétrico entre um eletrodo de carvão e uma peça a ser soldada [2].

Impulsionados pelo maior investimento em pesquisas, além do crescente desenvolvimento da informática e da robótica ao longo dos anos, foram sendo desenvolvidos novos processos de soldagem, como o processo de união com feixe de elétrons na França e na Alemanha em 1950 e a emissão da primeira patente de uma máquina de soldagem por ultrassom em 1960. Os avanços mais recentes, entretanto, são o desenvolvimento do processo de soldagem por fricção com pino não consumível (Friction Stir Welding) por 
Wayne Thomas e um time de pesquisadores do TWI (The Welding Institute), em 1991, e o processo Friction Hydro Pillar Processing (FHPP) em 1992 [3].

As aplicações industriais do processo Friction Stir Welding (FSW) na soldagem de materiais metálicos leves (com baixa temperatura de fusão), como ligas de alumínio e magnésio, são diversas e se concentram hoje principalmente nas indústrias aeronáuticas, automotivas, navais e aeroespaciais. Em contrapartida as aplicações dessa tecnologia na união de materiais com temperatura de fusão mais elevada, como ligas a base de cobre, ferro e níquel, são ainda escassas, pois a tecnologia está em desenvolvimento e existem limitações relacionadas aos materiais utilizados para fabricação das ferramentas e desgaste prematuro das mesmas [4].

Apesar da técnica de soldagem pelo processo FSW ter merecido destaque, sendo objeto de estudo em diversos centros de pesquisa tecnológica e Universidades do exterior, pouco se tem observado quanto a trabalhos realizados inteiramente no Brasil. Um dos motivos para isto certamente reside na necessidade de um alto investimento para a obtenção dos equipamentos específicos à soldagem FSW [5]. Além disso, apesar do processo FSW estar sendo objeto de estudo em vários centros de pesquisa no mundo, poucos estudam sua aplicação em robôs industriais, em que se destacam os institutos e as empresas: HGZ (Helmholtz-Zentrum Geesthacht), IWB e KUKA (em conjunto com o Grupo Airbus), na Alemanha; a ESAB, na Suécia; Institut Maupertuis e Institut de Soudure, na França; Cewac, na Bélgica; Friction Stir Link, nos Estados Unidos [6]. Neste contexto, o maior avanço tecnológico que se tem conhecimento é o desenvolvimento de uma tecnologia robotizada para a soldagem de alumínio e aço, aplicada na produção em série de um veículo pela Honda Motor Co [7,8].

Dessa forma, tem-se como objetivo desse projeto dimensionar um sistema de soldagem pelo processo Friction Stir Welding para adaptação em um robô industrial. Nesta etapa, foi realizado o dimensionamento dos eixos, mancais, porta-ferramentas, dentre outros dispositivos necessários ao correto funcionamento do sistema. Em seguida, foi necessário escolher o tipo de transmissão (corrente, correia, engrenagens) para fazer o acionamento do eixo principal. De modo geral, o sistema dimensionado deveria ter alto torque para suportar a soldagem de materiais com elevado ponto de fusão e peso reduzido, uma vez que seria adaptado a um robô industrial. Espera-se, desta forma, aumentar o ganho de conhecimento no que diz respeito à aplicação de um sistema de soldagem pelo processo Friction Stir Welding robotizado, buscando superar as barreiras ligadas à sua utilização em produtos manufaturados em larga escala, como será descrito nos próximos capítulos.

\subsection{Equipamento usados no processo FSW: estado da arte}

A literatura apresenta basicamente três tipos de equipamentos viáveis à execução de juntas soldadas pelo processo FSW:

- Máquinas ferramentas convencionais e fresadoras CNC's adaptadas [9-12];

- Máquinas dedicadas à soldagem pelo processo FSW [13,14];

- Robôs industriais adaptados [6,15,16].

Quando máquinas ferramentas, tais como fresadoras, são adaptadas para a soldagem, as mesmas devem ser reforçadas a fim de suportar as elevadas cargas envolvidas no processo e para aumentar a segurança dos operadores. Além disso, podem ser instrumentadas para obter um controle mais preciso do processo. Outras adaptações incluem a adição de motores para aumentar os graus de liberdade do equipamento, contribuindo assim com a flexibilidade do processo [17]. Almeida [9] utilizou uma fresadora CNC modelo Integrex e-TOWER 1060V do fabricante Mazak para a soldagem de ligas de alumínio pelo processo FSW, em que dispositivos e variáveis do processo foram adaptados para que a realização dos experimentos fosse viável, incluindo a geometria das ferramentas, os suportes e fixações, assim como alguns parâmetros de soldagem. As fresadoras CNC's adaptadas são viáveis para a utilização no processo FSW, uma vez que apresentam elevada rigidez, suportando assim as forças aplicadas durante a soldagem. Além disso, permitem uma grande variação de parâmetros por meio da inclinação do cabeçote, alta gama de rotação e velocidades de avanço e, ainda, possibilidade de monitoramento da força axial [18].

As máquinas dedicadas ao processo FSW são equipamentos projetados especificamente para tal finalidade, possuindo elevada rigidez, precisão e disponibilidade (Figura 1). São adequadas à soldagem de materiais de elevado ponto de fusão como aço, aço inoxidável, titânio, dentre outros. Por possuir alto grau de instrumentação, permite o controle de vários parâmetros inerentes ao processo. Geralmente estes equipamentos são de grande porte e 

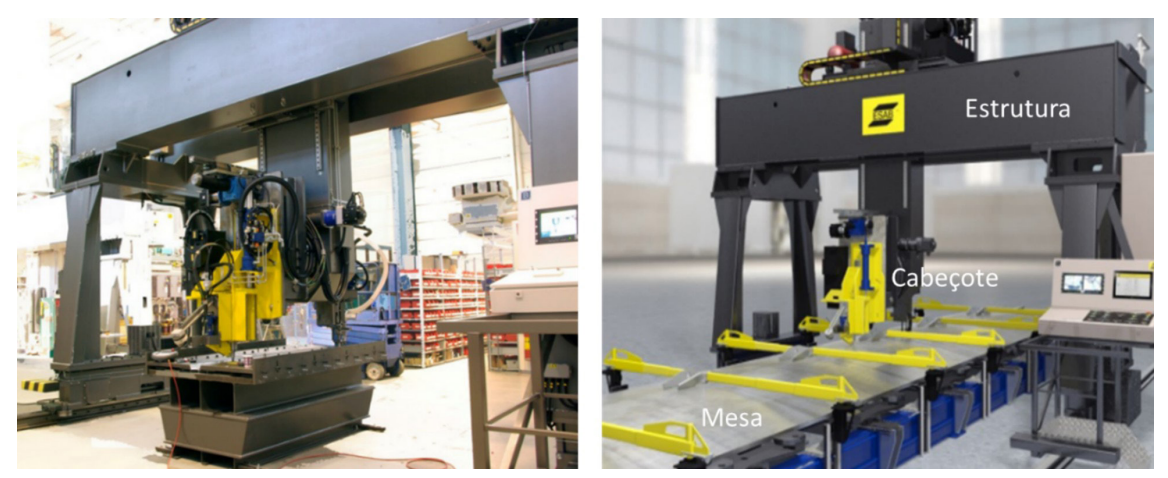

Figura 1. Equipamento dedicado a soldagem pelo processo Friction Stir Welding [16].

custo elevado, o que inviabiliza sua utilização em locais remotos. Embora tenha havido algum estudo sobre o dimensionamento de equipamentos portáteis dedicados ao processo $\mathrm{FSW}$, até o momento, não são conhecidos sistemas disponíveis comercialmente [17].

A primeira aplicação de um robô de soldagem pelo processo FSW foi apresentada ao público em Gotemburgo, Suécia, no ano 2000, durante o Friction Stir Welding Symposium por dois diferentes grupos de pesquisa [6]. Um grupo utilizava um robô antropomórfico modelo ABB IRB-6400 combinado a um motor elétrico de $220 \mathrm{~W}$ e o outro um robô Neos de hastes paralelas [15,19].

Os robôs industriais aplicados à soldagem pelo processo FSW possuem alta flexibilidade, podendo soldar componentes com geometrias complexas em escala industrial. Os desenvolvimentos recentes contribuíram para permitir a utilização dos robôs de braços articulados em série e robôs de braços paralelos, em que foram eliminados problemas de baixa capacidade de carga e falta de rigidez dos robôs. Mendes et al. [17] realizou uma comparação entre os principais equipamentos usados no processo de soldagem FSW disponíveis na literatura, comparando suas vantagens e desvantagens em diversos quesitos como flexibilidade, custo, possibilidade de soldagem de peças complexas, dentre outros.

\subsection{Adaptações de robôs industriais para a soldagem pelo processo FSW}

Estudos relacionados ao dimensionamento dos sistemas responsáveis pela movimentação da ferramenta não são encontrados facilmente na literatura. Mendes et al. [17] realizou o dimensionamento de um sistema de acionamento adaptado a um robô antropomórfico Motoman modelo ES165N com 6 graus de liberdade, que foi usado para a soldagem de polímeros pelo processo FSW (Figura 2). O sistema é composto por um conjunto de polias acionadas por um servo motor, em que os dados de entrada para os cálculos de dimensionamento foram: força axial de $4000 \mathrm{~N}$; rotação máxima de $1500 \mathrm{rpm}$; força transversal e radial de $2000 \mathrm{~N}$; torque de $4 \mathrm{~N} . \mathrm{m}$. Apesar da
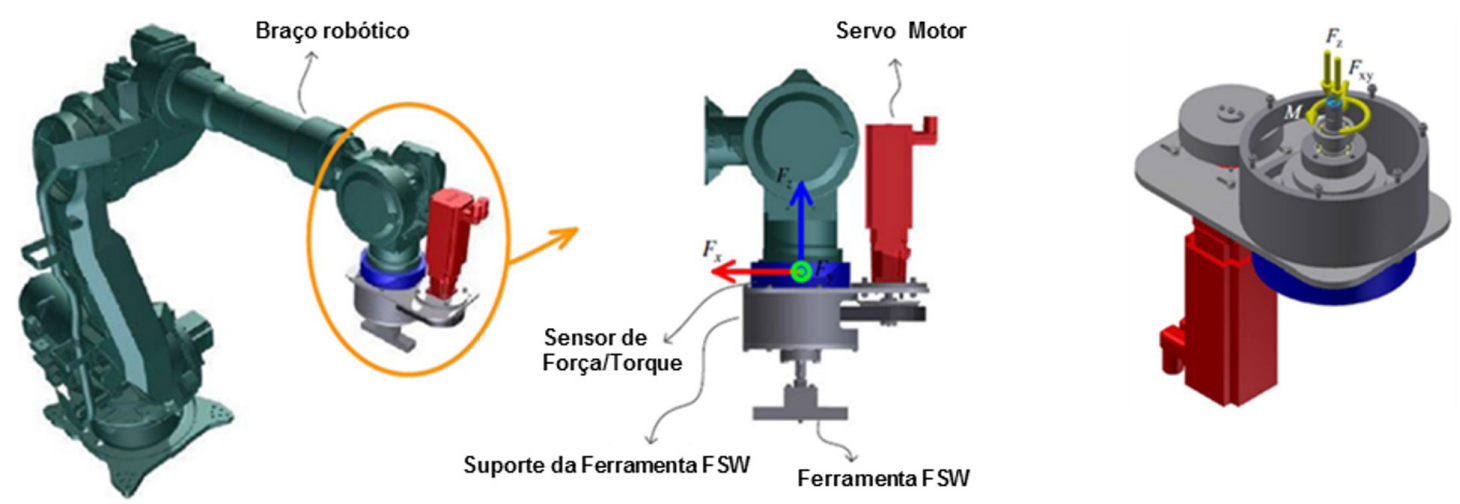

Figura 2. Modelagem 3D e identificação das forças envolvidas no processo de soldagem usando um robô Motoman modelo ES165N [17]. 
relevância do trabalho, o mesmo não pode ser usado como referência para o dimensionamento de um sistema para a soldagem de aços, em função dos altos valores de força envolvidos no processo, como será detalhado mais adiante.

Smith [15] apresenta suas tentativas e resultados ao adaptar um robô industrial para a soldagem FSW, em que foi escolhido o robô comercial ABB modelo IRB 6400, com 150 kg de capacidade. Para a adaptação do sistema de soldagem, o autor utilizou inicialmente um motor elétrico de $2200 \mathrm{~W}$, em que foram verificados problemas de vibração excessiva e ressonância nos braços do robô, bem como limitação de torque, que comprometiam a qualidade das soldas realizadas em chapas de alumínio 6061-T6 de 2 mm de espessura. Segundo o autor, o problema poderia ser mitigado ao utilizar posições de soldagem específicas em soldas de pequenos comprimentos. Para solucionar os problemas apresentados foi utilizado um motor hidráulico de 2200 W, com possibilidade de rotação até 3200 rpm, alimentado por uma bomba, conforme apresentado na Figura 3. Os resultados foram mais satisfatórios ao utilizar o motor hidráulico em comparação ao motor elétrico, sendo possível também reduzir a massa do sistema.

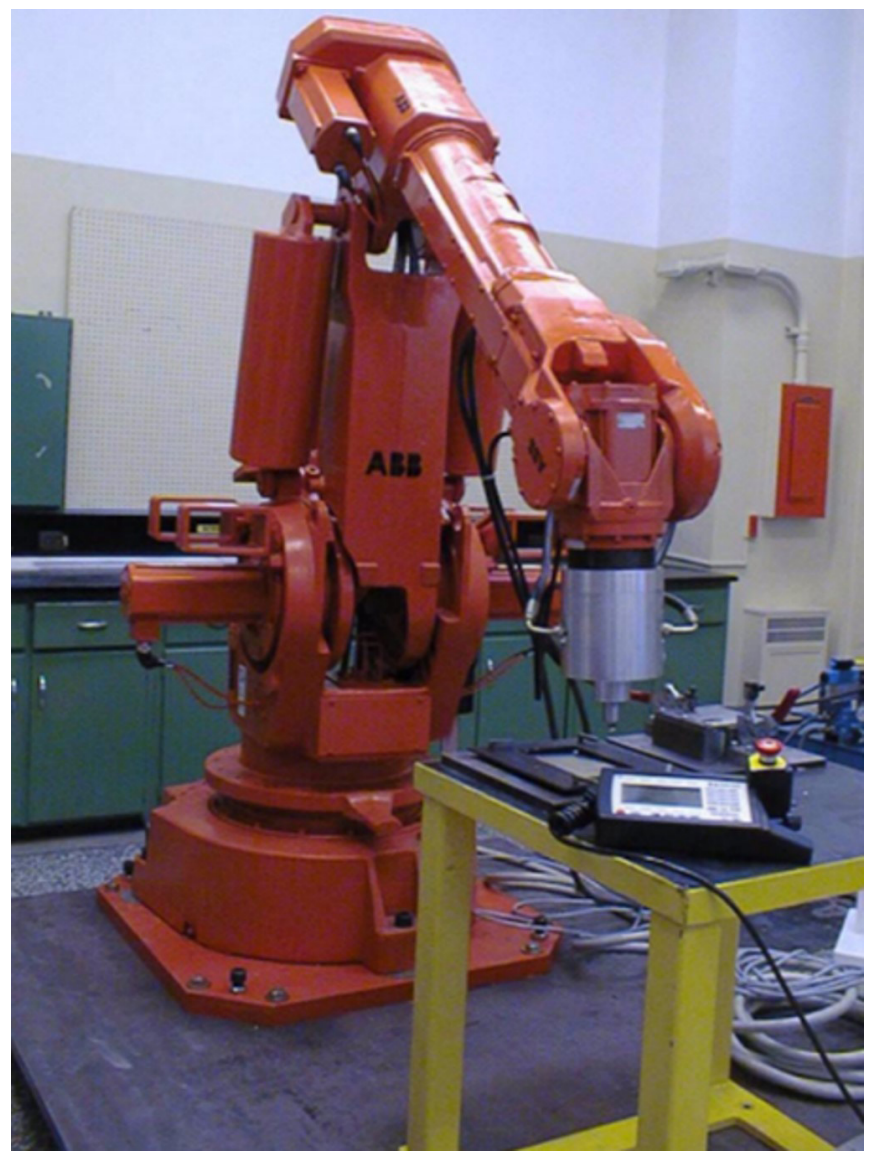

Figura 3. Robô ABB IRB 6400 adaptado a um motor hidráulico para a soldagem FSW [15].

A introdução de um hardware para controle das forças envolvidas no processo FSW é inviável em função dos custos e problemas de manutenção. Sendo assim, o autor implementou um software no robô para realizar os controles de força, já que a arquitetura do mesmo era aberta [15].

O CTP (Production Technology Centre) localizado em Trollhättan, na Suécia, é um centro de alta tecnologia que combina estudos nos processos de soldagem por laser, FSW, usinagem, conformação de materiais e realização de ensaios não destrutivos. Esse centro de pesquisa possui o sistema ESAB Rosio FSW, que conta com um robô da ABB modelo IRB-7600 com 6 graus de liberdade e capacidade de 500 kg. O último grau de liberdade do robô foi adaptado para permitir a instalação do sistema de soldagem, composto por um servo motor da Mavilor, modelo MAS-45, que permite rotações de até 4500 rpm e torque de 30 N.m (Figura 4). 


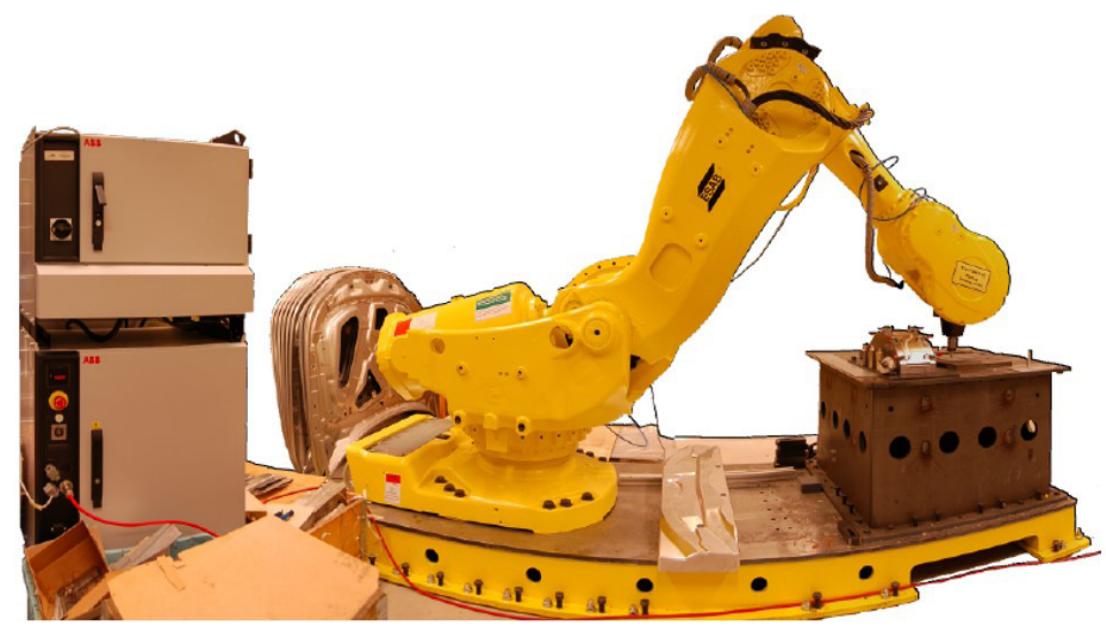

Figura 4. ESAB Rosio FSW [6].

O sistema ESAB Rosio FSW é, até o momento, o equipamento de soldagem FSW mais moderno apresentado pela literatura. O robô utilizado possui alta capacidade, e o sistema de soldagem é compacto e de baixo peso. Não foi possível encontrar dados referentes à caixa redutora de rotação do equipamento, entretanto, sabe-se que o servo motor ligado a ela possui massa de apenas $22 \mathrm{~kg}$, o que favorece a flexibilidade e eficiência do processo. Além disso, verificou-se que o ESAB Rosio FSW pode ser usado em processo de soldagem FSW híbrido, por meio do auxílio de um sistema a laser, com possibilidade, ainda, de utilização de um sistema de proteção por gases [15,16]. Uma das maiores vantagens do equipamento é a possibilidade de programação e simulação off-line, conforme apresentado na Figura 5.
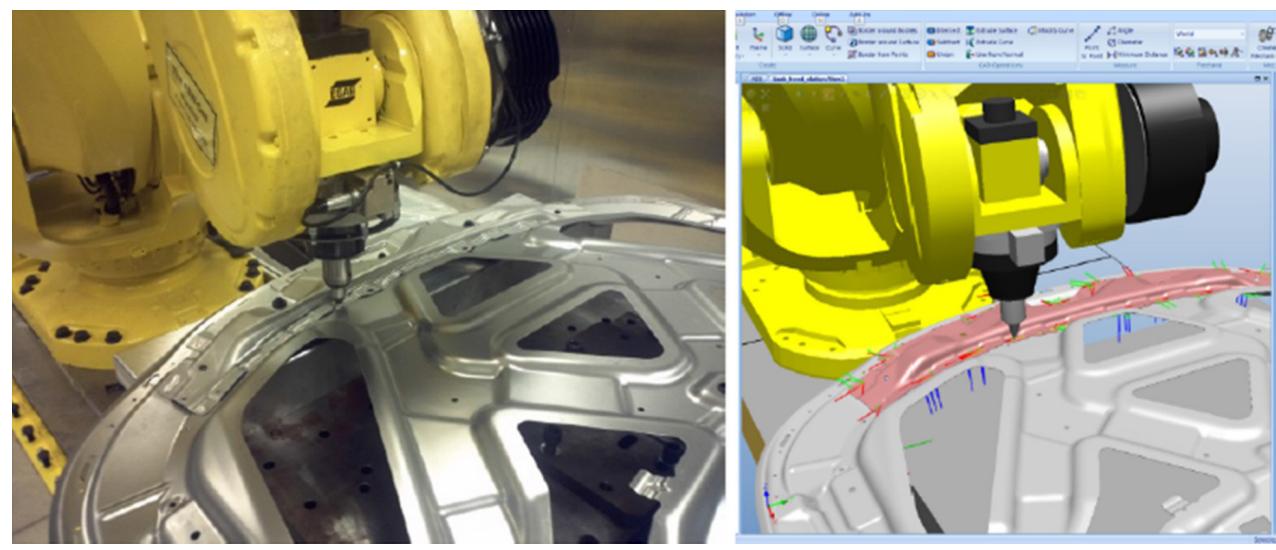

Figura 5. Soldagem de um componente em alumínio realizada por programação off line do processo [6].

A soldagem com sistema de visão artificial pode ser realizada por meio da aquisição de imagens feitas por uma câmera monocromática, modelo Prosilica EC650, com uma resolução de $640 \times 480$ pixels e uma taxa de quadros máxima de 90 fps (Figura 6). A câmara é conectada por meio de uma interface FireWire para a aquisição de dados no sistema LabVIEW e o processamento de imagens, por sua vez, é realizado em um modelo no software matemático MATLAB [6].

Backer [6] utilizou o ESAB Rosio FSW para a soldagem de ligas de níquel 718 (Haynes ${ }^{\circledR} 718$ ) com $2 \mathrm{~mm}$ de espessura, e para a soldagem de chapas de alumínio de alta resistência AA 7075-T6, de $2 \mathrm{~mm}$ de espessura. Posteriormente, Zhang [16] utilizou o sistema para a soldagem 3D de ligas de alumínio 6082-T6 com 1,5 mm de espessura. Nos dois casos, foi utilizado um software chamado ContRoSit para o controle e supervisão dos parâmetros 

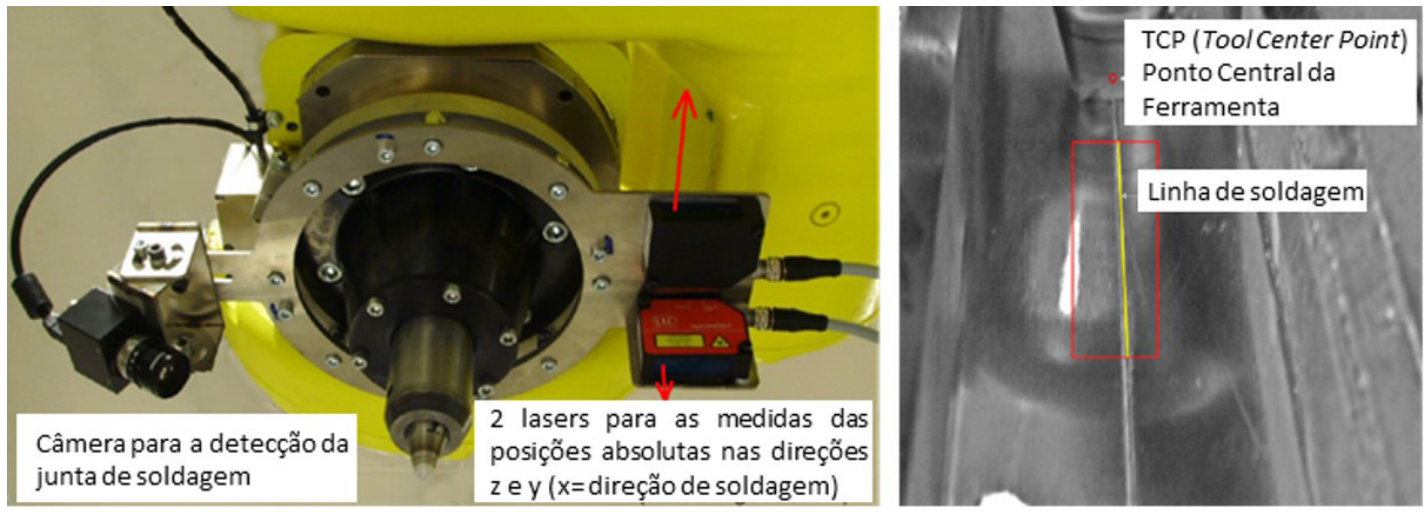

Figura 6. Soldagem de um componente após a aplicação do algoritmo de detecção do trajeto e montagem dos sensores e câmera para a identificação do percurso [6].

de soldagem do robô. As forças foram medidas usando o sistema de controle ABB's IRC5, que permitem avaliar as forças de compressão no sentido axial de até $18 \mathrm{kN}, 7,2 \mathrm{kN}$ nas direções planas e torque de até 1400 N.m.

Backer [6] afirma que é possível utilizar o sistema ESAB Rosio FSW assistido por laser para a soldagem de peças (Hybrid Friction Stir Welding). Entretanto, não foi encontrado na literatura a utilização do sistema supracitado para a soldagem de aço carbono e suas ligas.

Para a soldagem de ligas de níquel, Backer [6] fez a alteração da relação de engrenagens do sistema de soldagem de 2:3 para 2:1, em que foi possível aumentar o torque do sistema para até $90 \mathrm{~N} . \mathrm{m}$, e diminuir a velocidade de rotação para $1500 \mathrm{rpm}$. Tal medida permitiu chegar a um torque de $72 \mathrm{~N}$.m a uma velocidade de $900 \mathrm{rpm}$. Entretanto, não é possível identificar no trabalho os detalhes do sistema mecânico que permitiu a alteração supracitada.

Backer [6] cita que os detalhes da adaptação do sistema de soldagem para o robô podem ser encontrados na Tese de Soron [20]. Entretanto, esse trabalho não foi disponibilizado na rede de forma completa, podendo estar relacionado a questões de confidencialidade com a ESAB. No resumo de seu trabalho, Soron [20] apresenta que será desenvolvida uma metodologia para a soldagem 3D pelo processo $\mathrm{FSW}$, em que a implantação do protótipo inclui softwares para controle de movimentos da ferramenta, direção e forças envolvidas. A Figura 7 apresenta os componentes principais do sistema de soldagem do ESAB Rosio FSW, composto por um servo motor da Mavilor, modelo MAS-45, e um redutor de especificação desconhecida.

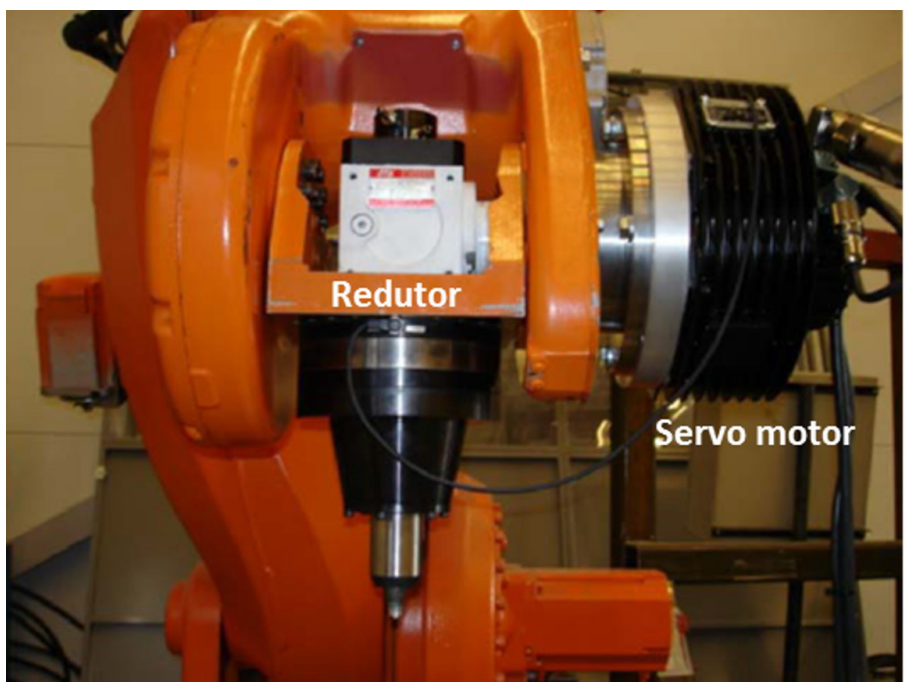

Figura 7. ESAB Rosio FSW, evidenciando os principais componentes do sistema de soldagem [20]. 
Esab [21] apresenta as características técnicas e dimensionais do ESAB Rosio FSW, em que se verifica um torque máximo de $44 \mathrm{~N} . \mathrm{m}$, rotação máxima de $3000 \mathrm{rpm}$ e avanços superiores a $1000 \mathrm{~mm} / \mathrm{min}$. Esses dados servem de referência para o dimensionamento de um sistema de soldagem FSW robotizado, entretanto, deve-se atentar que as os valores de torque e velocidade do sistema de soldagem do robô limita sua utilização à união de materiais com baixo ponto de fusão, como alumínio.

A contribuição mais recente encontrada na literatura para sistemas robotizados aplicados ao processo FSW é da empresa Honda, que foi a primeira empresa do ramo automotivo a desenvolver uma tecnologia robotizada capaz de unir aço com alumínio, utilizado na produção em série do veículo Honda Accord 2013 [7].

\subsection{Reações relevantes ao processo Friction Stir Welding}

As reações mais relevantes geradas no processo FSW são o torque, a força na direção da soldagem e a força na direção transversal à junta soldada. $O$ torque resultante na junta é governado pelas características de fluxo dos materiais a serem soldados, e pela velocidade do fuso. Dessa forma, a menor força axial, combinada com maior velocidade de rotação e menor velocidade de avanço resulta em menor torque na junta soldada, pois proporciona maior entrada de calor, o que aumenta a fluidez do material [22].

Santos et al. [22] soldaram aços inoxidáveis duplex e super duplex de $6 \mathrm{~mm}$ de espessura, usando um equipamento Technologies Inc. (TTI), dedicado à soldagem pelo processo FSW. Para as soldagens foi utilizada uma ferramenta de PCBN-40\% W-Re com ombro de $25 \mathrm{~mm}$ de diâmetro e pino com comprimento de $6 \mathrm{~mm}$ e $8 \mathrm{~mm}$ de diâmetro. Verifica-se na Tabela 1 as forças e torques envolvidos no processo, em que a força máxima no eixo

Tabela 1. Variáveis de saída durante a soldagem dos aços duplex e super duplex [22].

\begin{tabular}{|c|c|c|c|c|c|c|c|}
\hline \multirow{2}{*}{ UNS } & \multicolumn{2}{|c|}{$\begin{array}{l}\text { Força na direção axial } \\
\qquad(\mathbf{k N})\end{array}$} & \multicolumn{2}{|c|}{$\begin{array}{l}\text { Força na direção de } \\
\text { soldagem (kN) }\end{array}$} & \multirow{2}{*}{$\begin{array}{l}\text { Torque médio } \\
\text { (N.m) }\end{array}$} & \multirow{2}{*}{$\begin{array}{c}\mathrm{HI} \\
(\mathrm{kJ} / \mathrm{mm})\end{array}$} & \multirow{2}{*}{$\begin{array}{c}\text { Máxima } \\
\text { temperatura na } \\
\text { ferramenta FSW } \\
\left({ }^{\circ} \mathrm{C}\right)\end{array}$} \\
\hline & Média & Máximo & Média & Máximo & & & \\
\hline S32101 & $37,0 \pm 0,2$ & 37,5 & $4,8 \pm 0,7$ & 6,6 & $118 \pm 2$ & 1,48 & 856 \\
\hline S3205 & $36,9 \pm 0,3$ & 37,5 & $3,3 \pm 1,1$ & 6,6 & $119 \pm 5$ & 1,50 & 844 \\
\hline S32750 & $37,0 \pm 0,2$ & 37,4 & $7,4 \pm 0,8$ & 9,3 & $108 \pm 1$ & 1,36 & 818 \\
\hline S32760 & $37,0 \pm 0,2$ & 37,9 & $6,7 \pm 1,3$ & 9,5 & $110 \pm 4$ & 1,38 & 815 \\
\hline
\end{tabular}

"z" foi de 37,9 kN e a força máxima na direção de avanço foi de 9,5 kN. O torque máximo, por sua vez, foi de 119 N.m a aproximadamente $200 \mathrm{rpm}$.

Kozminski [13] fez a soldagem do aço inoxidável duplex UNS S31803 de $4 \mathrm{~mm}$ de espessura utilizando um equipamento dedicado ao processo FSW chamado Gantry System, que possui mesa com deslocamento horizontal, carga axial máxima de $80 \mathrm{kN}$, velocidade de avanço entre 1 e $160 \mathrm{~mm} / \mathrm{s}$ e rotação máxima de $3500 \mathrm{rpm}$. Foi utilizada uma ferramenta com ombro de $36,8 \mathrm{~mm}$ e pino cônico roscado com comprimento de $3,7 \mathrm{~mm}$ e diâmetro maior de 9,2 mm. Durante os testes, foi utilizada velocidade de rotação e força axial constantes de $500 \mathrm{rpm}$ e $45 \mathrm{kN}$, respectivamente, variando a velocidade de avanço entre 1, 2, 3 e $4 \mathrm{~mm} / \mathrm{s}$. Encontrou-se valores de torque médio entre 53 e $73 \mathrm{~N} . \mathrm{m}$, sendo o primeiro valor referente à velocidade de $1 \mathrm{~mm} / \mathrm{s}$ e o segundo valor para a velocidade de $4 \mathrm{~mm} / \mathrm{s}$.

Arora et al. [23] fez uma simulação dos valores de torque em função da velocidade de rotação da ferramenta durante a soldagem de ligas de alumínio AA2524 de 6,4 mm de espessura, com velocidade de avanço de $2,11 \mathrm{~mm} / \mathrm{se}$ força axial constante de 42,3 kN. A ferramenta, por sua vez, apresentava ombro com diâmetro de $20,3 \mathrm{~mm}$ e pino de 6,2 $\mathrm{mm}$ de comprimento e 7,1 mm de diâmetro. Os resultados das simulações, comparadas aos resultados experimentais de Yan et al. [24], apresentados na Figura 8.

Husain et al. [25] soldou o aço SAE J412 de $2 \mathrm{~mm}$ de espessura por meio de um equipamento dedicado à soldagem pelo processo FSW (RM Series; RM1A-0.7), usando uma ferramenta de carboneto de tungstênio com diâmetro do ombro de $36,8 \mathrm{~mm}$ e pino de $1,8 \mathrm{~mm}$ de comprimento e $5 \mathrm{~mm}$ de diâmetro. Os parâmetros de soldagem, bem como o torque resultante em função da variação da rotação usada são apresentados na Tabela 2. 


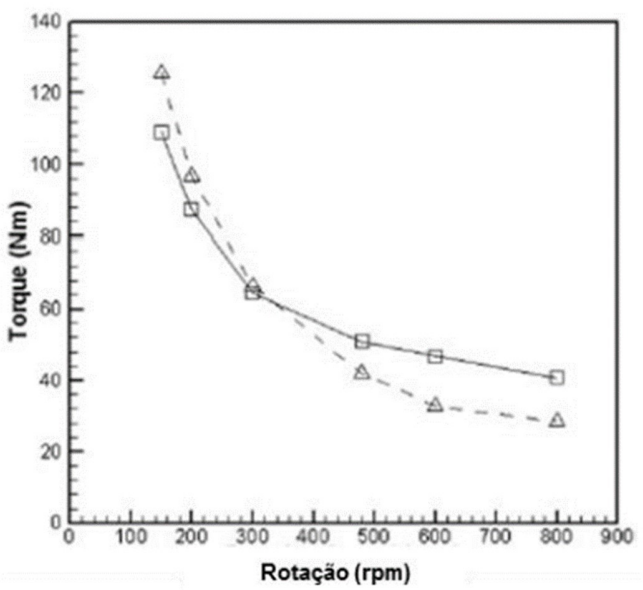

Figura 8. Torque em função da rotação na soldagem da liga AA2524: resultados da simulação (linha cheia) e resultados experimentais (linha tracejada) [23].

Tabela 2. Dimensões da ferramenta e parâmetros de soldagem [25].

\begin{tabular}{|c|c|c|c|c|c|c|c|c|c|c|}
\hline \multicolumn{6}{|c|}{ Dimensões da ferramenta } & \multirow[b]{2}{*}{ 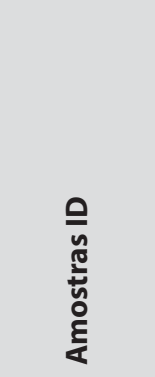 } & \multicolumn{4}{|c|}{ Parâmetros de soldagem } \\
\hline 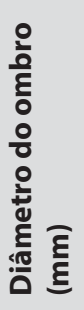 & 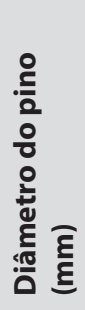 & 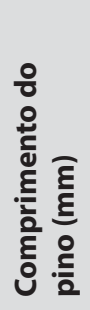 & 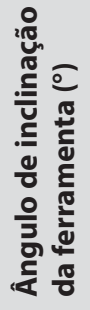 & 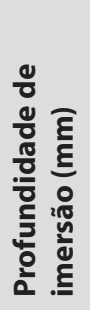 & 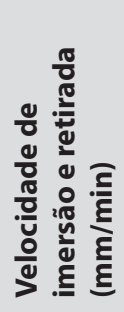 & & 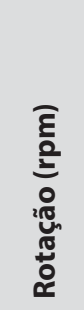 & 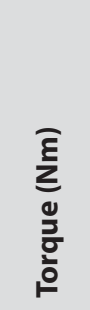 & 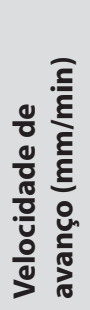 & 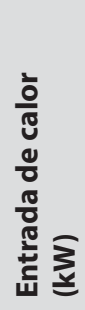 \\
\hline \multirow{4}{*}{36,8} & \multirow{4}{*}{5} & \multirow{4}{*}{1,8} & \multirow{4}{*}{1,5} & \multirow{4}{*}{1,9} & \multirow{4}{*}{0,6} & Amostra 1 & 800 & 84,5 & \multirow{4}{*}{50} & 56,3 \\
\hline & & & & & & Amostra 2 & 1000 & 89,8 & & 69,9 \\
\hline & & & & & & Amostra 3 & 1200 & 97,8 & & 83,2 \\
\hline & & & & & & Amostra 4 & 1400 & 104,7 & & 98,2 \\
\hline
\end{tabular}

Lienert et al. [26] realizaram a soldagem do aço AISI 1018 de 6,35 mm de espessura usando uma ferramenta com elementos de liga molibdênio e tungstênio, com diâmetro do ombro de $19 \mathrm{~mm}$ e pino com 6,22 mm de comprimento. Foi utilizado um sistema de aquisição de dados para verificação em tempo real do carregamento, torque e temperatura envolvidos no processo. Os valores de torque e carga durante o processo são apresentados na Figura 9, em que a rotação esteve entre 450 e 650 rpm. O período de imersão da ferramenta durou quase três minutos, sendo que a carga subiu inicialmente quando o pino tocou a chapa à temperatura ambiente, e caiu rapidamente à medida que esta foi aquecendo e amaciando o material. Esse ciclo se repetiu à medida que o pino penetrava na chapa e encontra novamente uma região mais fria (Figura 9A). O pico de carga se dá a aproximadamente $175 \mathrm{~s}$ do início do processo de imersão a um valor de $3300 \mathrm{kPa}$ (7600 lbf), referente ao momento em que o ombro da ferramenta entra em contato com as chapas a serem soldadas. À medida que o ombro da ferramenta toca as chapas, o valor do torque aumenta e fica constante em cerca de 56,5 N.m (500 In.lbs) durante todo o período de soldagem. Nenhuma mudança apreciável foi encontrada no torque com diferentes velocidades de deslocamento.

As cargas axiais médias ficaram na faixa de 4,700 lbf (18,7 kN), quando utilizou-se velocidades de 1 pol./min (Figura 9B). Tem-se o aumento das cargas axiais com o aumento da velocidade de avanço. Os valores de torque e carga durante a soldagem do aço AISI 1018 podem ser comparados à soldagem de outros materiais como Ti e Al [26]. De acordo com os autores, o torque encontrado foi menor do que aquele verificado na soldagem de uma liga de Ti-6 Al-4 V (675 polegadas-lb) e os valores eram comparáveis à soldagem de uma liga Al 7075-T6 pelo processo FSW. Entretanto, os valores encontrados durante a soldagem do aço foram maiores do que aqueles identificados na soldagem das ligas de Al 5083-O, 6082-T6 e 6061-T6. 

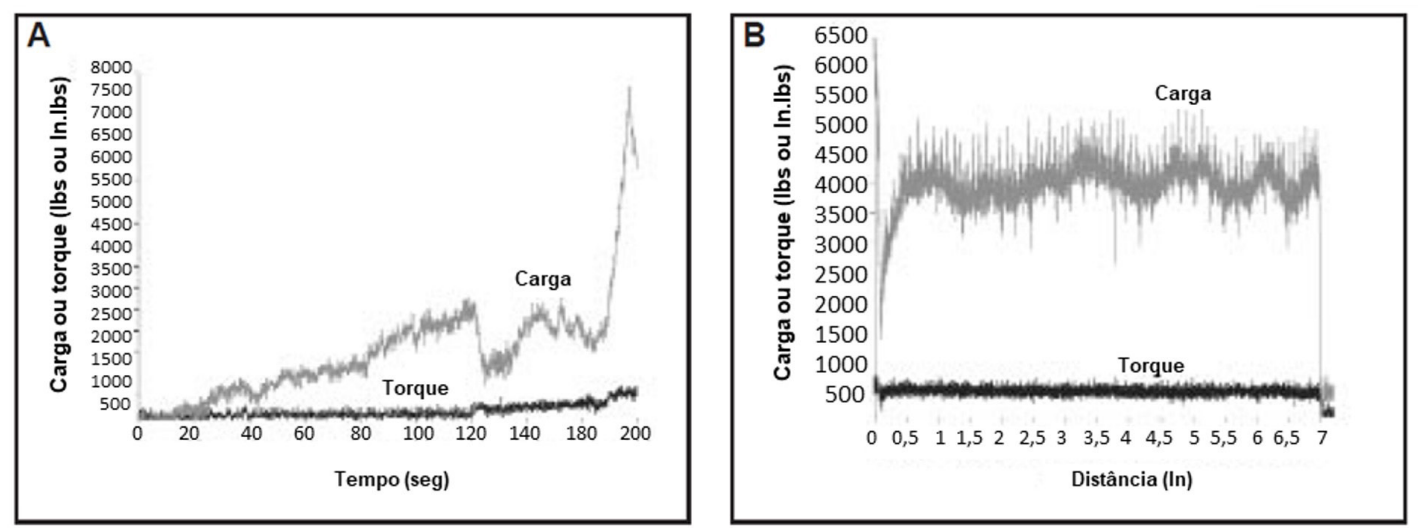

Figura 9. Curva de carregamento e torque em função do tempo e distância de soldagem: (A) período de imersão da ferramenta; (B) durante a translação da ferramenta [27].

\section{Materiais e Métodos}

O dimensionamento do sistema de soldagem foi baseado, principalmente, na revisão bibliográfica, em que foram analisadas as reações mais relevantes durante a soldagem pelo processo FSW, sendo elas: o torque, a força na direção da soldagem e a força na direção transversal à junta soldada. Nesta etapa, foi realizado o dimensionamento dos eixos, mancais, porta-ferramentas, dentre outros dispositivos necessários ao correto funcionamento do sistema de soldagem. Em seguida, foi necessário escolher o tipo de transmissão (corrente, correia, engrenagens) para fazer o acionamento do eixo principal. De modo geral, o sistema dimensionado deveria ter alto torque, para suportar a soldagem de materiais com elevado ponto de fusão e peso reduzido, uma vez que seria adaptado ao robô antropomórfico COMAU NJ500, com 6 graus de liberdade e capacidade de $500 \mathrm{~kg}$.

\section{Resultados e Discussão}

Para dimensionar o sistema de soldagem, verificou-se por meio da literatura as forças envolvidas no processo de união de diversos materiais, sobretudo, daqueles dotados de elevada temperatura de fusão, que tornam necessários maiores valores de força e torque. As reações mais relevantes geradas no processo FSW são o torque, a força na direção da soldagem e a força na direção transversal à junta soldada.

Com base nos valores de carga e torque apresentados pela literatura, escolheu-se um sistema moto redutor para acionamento do sistema, uma vez que este permite obter maior torque e resistência mecânica, comparado aos sistemas de acionamento por correia ou servo motores. Sendo assim, escolheu-se um redutor comercial modelo GD49, obtido por meio de uma parceria com a empresa Geremia Redutores.

O redutor aplicado a este projeto possui mancal axial com rolamento autocompensador de rolos, específico para suportar cargas exercidas pelas extrusoras. Essa classe de redutor tem baixo custo de instalação devido ao seu tamanho compacto e a transmissão ao engrenamento ser feita diretamente pelo motor, não sendo necessário órgão auxiliar. A transmissão do redutor é feita por engrenagens cilíndricas de dentes helicoidais, o que proporciona um funcionamento silencioso e constante [27]. O motor utilizado possui tensão de $220 \mathrm{~V}$ e é do tipo industrial com grau de proteção IP55, se enquadrando nas leis de eficiência energética que entrou em vigor em janeiro de 2010.

O sistema de soldagem dimensionado é apresentado na Figura 10, em que é possível identificar o braço robótico (A) unido ao dispositivo de soldagem dimensionado (C) por meio de um acoplamento (B). A redução escolhida para este projeto foi a de 1:5,69, que proporciona torque de 282 N.m a 298,8 rpm, e torque máximo de 650 N.m (Figura 10C). O motor elétrico usado no acionamento do redutor permite rotação máxima de 1700 rpm, sendo de $4 \mathrm{P}$ e $60 \mathrm{~Hz}$ (Figura 10D).

Os detalhes do eixo de saída do sistema projetado (Spindle) são apresentados na Figura 11. Sabe-se que o redutor GD49 foi concebido para suportar carga axial máxima de $120 \mathrm{kN}$, tendo uma baixa resistência a cargas radiais. Sendo assim, fez-se necessário dimensionar um mancal auxiliar, de modo que o sistema pudesse suportar 
as cargas radiais impostas durante o processo FSW (Figura 11A). O mancal auxiliar foi dimensionado em aço SAE 1045 e possui um rolamento de contato angular 3210B de capacidade de carga dinâmica de 51 kN (Figura 11B) e um rolamento $6210 Z Z$ de capacidade de carga dinâmica de 35 kN (Figura 11C), separados por um espaçador fabricado em SAE 64 (Figura 11D).

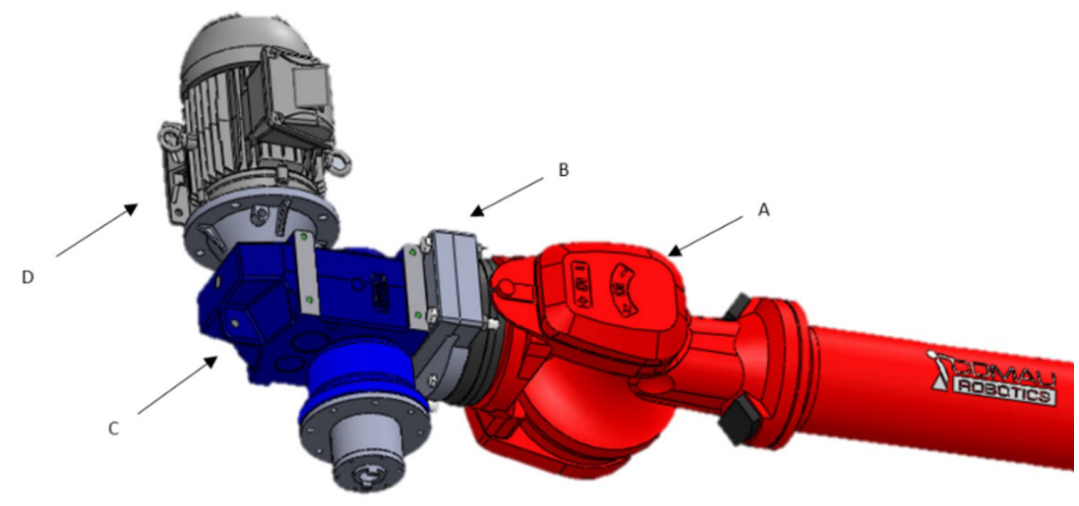

Figura 10. Sistema de soldagem projetado: (A) braço robótico; (B) acoplamento; (C) dispositivo de soldagem; (D) motor elétrico.

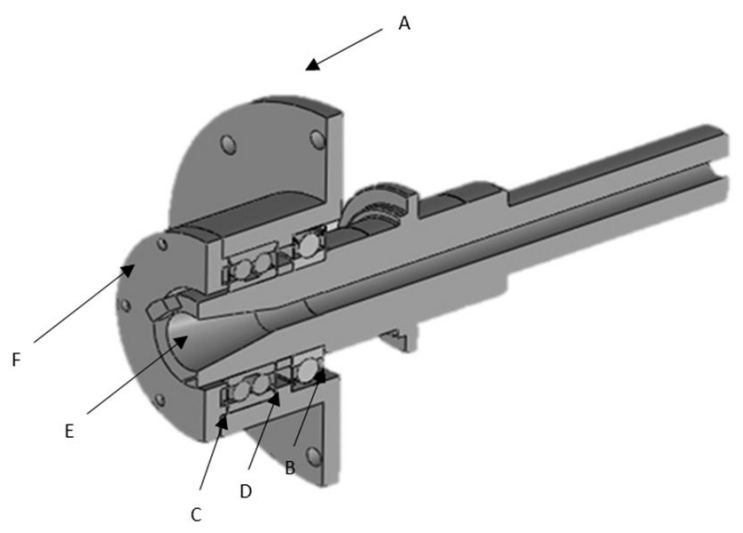

Figura 11. Detalhes do eixo de saída (Spindle) e mancal auxiliar.

O eixo de saída, por sua vez, teve suas dimensões adaptadas para permitir a fixação de um porta ferramentas do Tipo Weldon (Figura 11B), sendo o cone e a fixação dimensionados conforme a norma ANSI B5.18 (Figura 11E). A fixação Weldon foi escolhida por permitir alta segurança contra a extração da ferramenta, e por ser a ideal em processos de transmissão de torque. Além disso, a fixação Weldon possui menor custo, comparada às fixações hidráulicas e térmicas.

O eixo de saída padrão do redutor GD49 é fabricado em aço SAE 1045, entretanto, para suportar os esforços decorrentes da soldagem foi alterado para o material SAE 4340. A tampa do mancal auxiliar foi dimensionada em aço SAE 1020 e tem a função de manter o rolamento auto compensador acoplado ao alojamento do mancal, bem como impedir que materiais e impurezas penetrem no interior do sistema e comprometam seu funcionamento (Figura 11F).

Verifica-se na Figura 12 o esquema do processo de montagem do porta ferramentas do tipo Weldon (B) ao sistema de soldagem dimensionado (A), bem como a montagem da ferramenta de soldagem (C) ao porta ferramentas supracitado. O tirante (Figura 12D) é um componente estrutural que é montado pelo lado posterior do redutor e passa por dentro do eixo de saída (Figura 12E), sendo este responsável por fixar o porta ferramentas (Figura 12B) por meio de uma de rosca M12. 


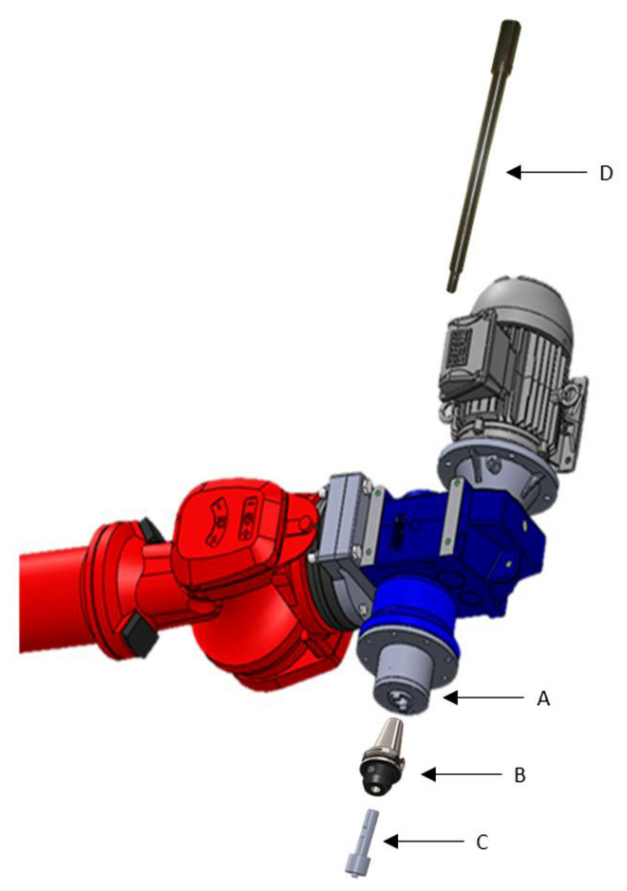

Figura 12. Montagem da ferramenta ao sistema de soldagem: (A) dispositivo de soldagem; (B) porta ferramentas Weldon; (C) ferramenta para a soldagem; (D) tirante.

A montagem experimental realizada é apresentada na Figura 13, em que é possível verificar o robô antropomórfico COMAU NJ500 de 6 graus de liberdade (A) que foi usado para a adaptação do sistema de soldagem dimensionado (B). A base de soldagem é a mesa de uma furadeira de coluna adaptada como estrutura para a fixação das chapas a serem unidas pelo processo FSW (Figura 13C). Para fazer o controle da rotação do sistema de soldagem foi utilizado um inversor de frequência com capacidade de acionamento de motores de até $50 \mathrm{cv}$, conforme apresentado na Figura 14.

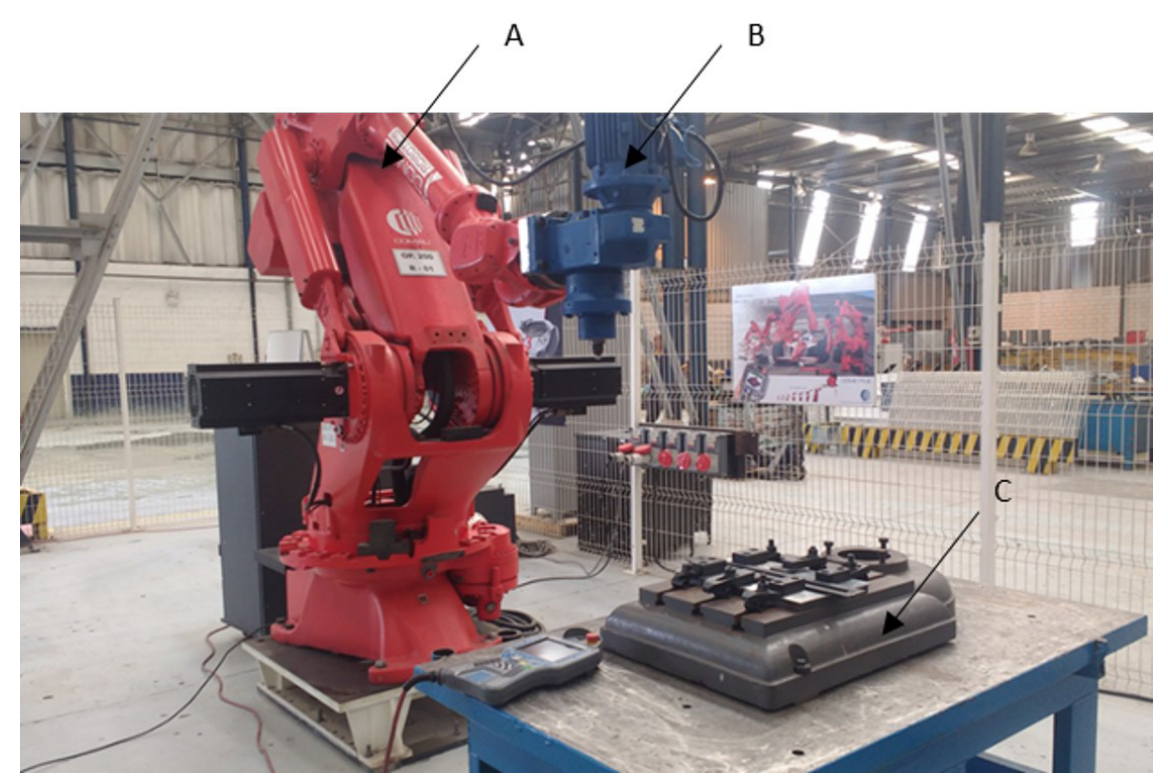

Figura 13. Montagem experimental: (A) robô antropomórfico COMAU NJ500; (B) dispositivo de soldagem; (C) base de soldagem. 

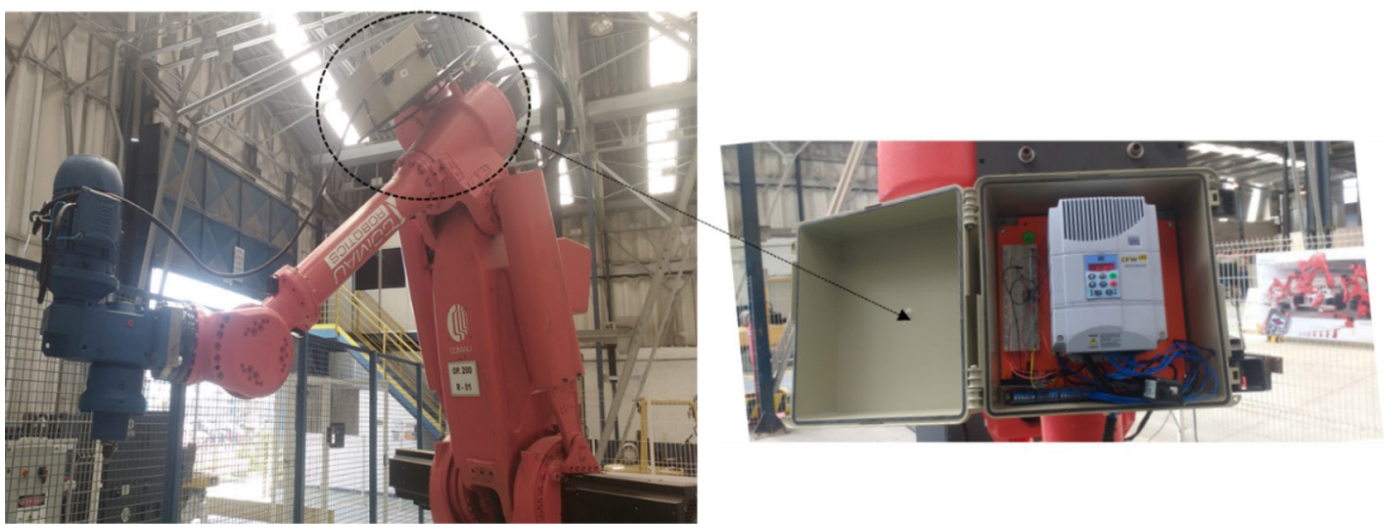

Figura 14. Inversor de frequência acoplado ao robô.

Após a montagem do sistema, o mesmo foi usado para testes durante a soldagem da liga de alumínio 5052 H34, em que a velocidade rotacional da ferramenta foi mantida constante em 378 rpm, e a velocidade de avanço em 7,5 mm/min. A rotação foi medida experimentalmente por meio de um tacômetro digital modelo DT-2234C, com resolução de 0,1 rpm e precisão de $\pm 0,05 \%+1$ dígito. Para o teste definitivo a ferramenta sofreu uma inclinação de $2^{\circ}$, e o tempo de espera (dwell time) antes de iniciar o translado da ferramenta foi de $20 \mathrm{~s}$. Foram utilizadas ferramentas fabricadas em aço AISI H13, com pino cônico liso e ombro de 18 mm de diâmetro.

Verifica-se na Figura 15 as imagens em vista de topo das soldas realizadas nas chapas de alumínio 5052 H34 com a ferramenta usada (A) e com a ferramenta nova (B). Verificou-se por meio de um paquímetro que a largura do cordão de solda correspondeu em toda sua extensão ao diâmetro do ombro do pino, evidenciando que não houve vibração dos braços do robô durante o processo. Com os parâmetros usados, foi possível obter soldas com bom aspecto superficial, ausência de rebarbas e penetração total.

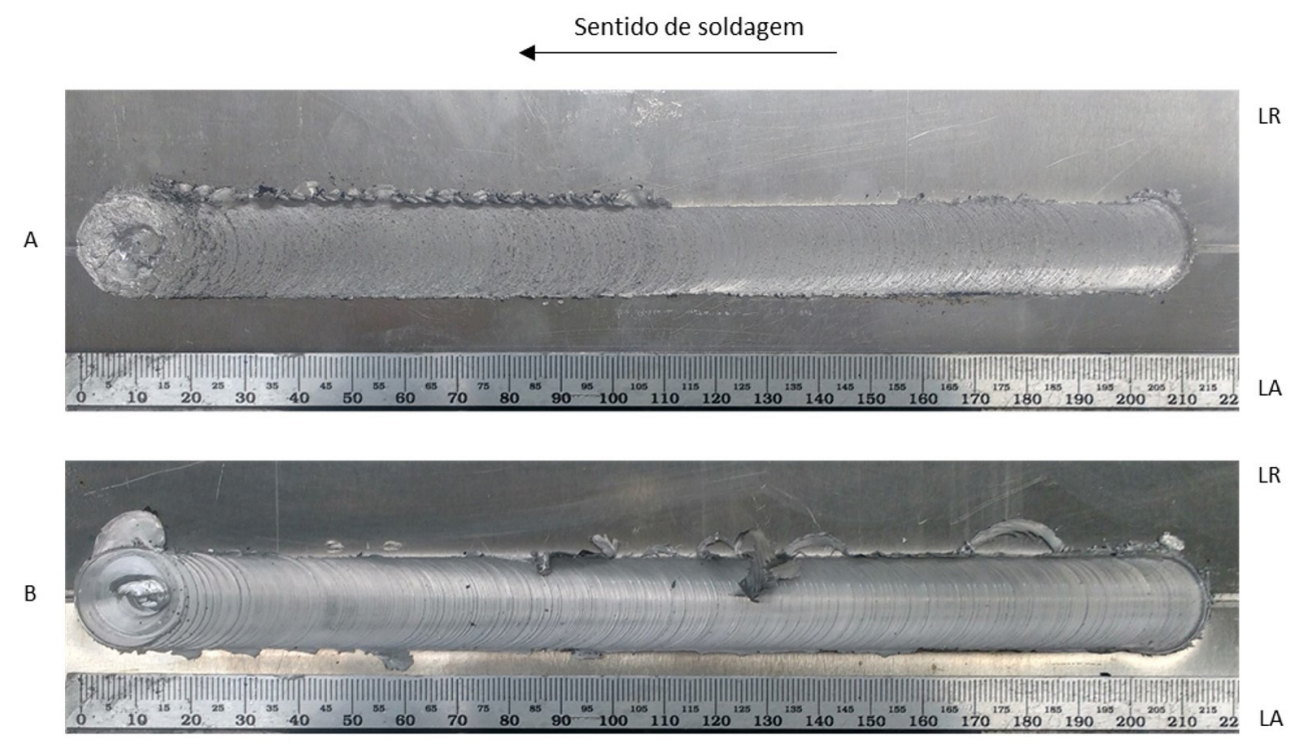

Figura 15. Soldas de topo realizadas nas chapas de alumínio 5052 H34 com a ferramenta usada (A) e com a ferramenta nova (B).

As próximas etapas deste projeto envolvem a caracterização do material soldado por meio de testes destrutivos de tração, dobramento, dureza e metalografia. Em seguida, será realizada a soldagem dissimilar do aço SAE 1020 à liga de alumínio 5052 H34. 


\section{Conclusões}

Este trabalho apresentou o estado da arte sobre a utilização do processo de soldagem FSW robotizado, em que foi possível identificar as adaptações realizadas por diversos pesquisadores, até se chegar aos atuais sistemas: soldagem FSW robotizado com programação off line; sistemas com reconhecimento automático do caminho de soldagem; desenvolvimento da primeira tecnologia robotizada capaz de solda aço com alumínio pelo processo FSW, aplicado na produção em série de um veículo.

Além disso, foi possível identificar alguns trabalhos que evidenciam as reações de força axial, força transversal, torque na soldagem de diversos materiais e dimensões das ferramentas, que serviram de base para o dimensionamento de um sistema capaz de soldar materiais com elevado ponto de fusão. Salienta-se que o sistema apresentado neste trabalho está em processo de pedido de patente.

O sistema de soldagem robotizado desenvolvido foi testado durante a soldagem da liga de alumínio 5052 H34, em que foi possível obter soldas com bom aspecto superficial, ausência de rebarbas e penetração total. Os estudos serão continuados, de modo a realizar a caracterização das juntas soldadas por meio dos testes destrutivos de tração, dobramento, dureza e metalografia. Em seguida, será realizada a soldagem dissimilar do aço SAE 1020 à liga de alumínio 5052 H34.

\section{Agradecimentos}

Ao LRSS (Laboratório de Robótica Soldagem e Simulação) da UFMG e à CAPES (Coordenação de Aperfeiçoamento de Pessoal de Nível Superior), pelo apoio e investimento à pesquisa. À COMAU do Brasil pela parceria e empréstimo do robô SMART NJ500, indispensável à essa pesquisa. A empresa Geremia Redutores, pelo apoio à pesquisa por meio da doação do redutor GD49, destinado à adaptação ao sistema de soldagem.

\section{Referências}

[1] Modenesi PJ, Marques PV, Santos DB. Metalurgia da soldagem. Belo Horizonte: Laboratório de Soldagem e Ensaios Não Destrutivos, UFMG; 2001.

[2] Marques PV, Modenesi PJ, Bracarense AQ. Soldagem: fundamentos e tecnologia. Belo Horizonte: Editora da UFMG; 2005.

[3] Xiong J, Yang X, Lin W, Liu K. Microstructural characteristics and mechanical heterogeneity of underwater wet friction taper plug welded joints for low-alloy pipeline steel. Materials Science And Engineering: A. 2017;695: 279-290.

[4] Ramirez AJ, Benati DM, Fals HC. Effect of tool offset on dissimilar CU-AISI 316 stainless steel friction stir welding. In: International Society of Offshore and Polar Engineers. Proceedings of the 21st International Offshore and Polar Engineering Conference; 2011 June 19-24; Maui, Hawaii, USA. Hawaii: ISOPE; 2011.

[5] Capelari TV. Avaliação da geometria de ferramenta e parâmetros do processo FSW na soldagem da liga de alumínio AA 5052 [dissertação de mestrado]. Porto Alegre: Universidade Federal do Rio Grande do Sul; 2006.

[6] Backer J. Feedback control of robotic friction stir welding [Phd thesis]. Sweden: University West; 2014.

[7] Almeida DT. Análise microestrutural e avaliação mecânica de juntas soldadas por fricção e mistura mecânica (FSW) da liga de alumínio 5182-O [dissertação de mestrado]. Porto Alegre: Universidade Federal do Rio Grande do Sul; 2015.

[8] Gibson BT. The development of in-process monitoring and control techniques for aerospace applications of Friction Stir Welding [doctor thesis]. Nashville: Vanderbilt University, 2015.

[9] Almeida DT. Estudo dos parâmetros no processo de soldagem de mistura por atrito (FSW) da liga de alumínio 5083-O [trabalho de conclusão de curso]. ljuí: Universidade Regional do Noroeste do Estado do Rio Grande do Sul; 2013.
[10] Hiller DJ. Estudo de parâmetros e percursos no processo de soldagem de mistura por atrito (FSW) da liga de alumínio 5052 [dissertação de mestrado]. Rio de Janeiro: Universidade Federal do Rio de Janeiro; 2007.

[11] Longhurst WR. Force control of friction stir welding [doctor thesis]. Nashville: Vanderbilt University; 2009.

[12] López EAT. Simulación computacional de la transferencia de calor en herramientas usadas en soldadura por fricción-agitación. Revista UIS Ingenierías. 2016;14(2).

[13] Kozminski ELO. Análise microestrutural e avaliação mecânica de juntas soldadas por fricção e mistura mecânica (FSW) do aço inoxidável duplex UNS S31803 [dissertação de mestrado]. Porto Alegre: Universidade Federal do Rio Grande do Sul; 2015.

[14] ESAB. Rosio ${ }^{\mathrm{TM}}$ : Friction Stir Welding Robot for welding of challenging joints. 2016 [acesso em 30 jun. 2016]. Disponível em: http://esabsp.esab.net/files/Fact\%20sheets/Welding\%20 Automation/XA00133620.pdf.

[15] Smith CB. Robotic friction stir welding using a standard industrial robot. Journal of Light Metal Welding and Construction. 2004;42(3):40-41.

[16] Zhang C. Robotic 3D friction stir welding: T-butt joint [master thesis]. Sweden: University West; 2015.

[17] Mendes N, Neto P, Loureiro A, Moreira AP. Machines and control systems for friction stir welding: a review. Materials \& Design. 2016;90:256-265.

[18] Cook GE, Smartt HB, Mitchell JE, Strauss AM, Crawford R. Controlling robotic friction stir welding. Welding Journal. 2003;82(6):28-34.

[19] Von Strombeck A, Schilling C, Santos JF. Robotic friction stir welding-tool, technology and applications. Biuletyn Instytutu Spawalnictwa (Poland). 2001;45(6):49-52. 
[20] Soron M. Robot system for flexible 3D friction stir welding [doctor thesis]. Örebro: Örebro University; 2007.

[21] ESAB. The SuperStir ${ }^{\mathrm{TM}}$ FSW Gantry. 2016 [acesso em 30 jun. 2016]. Disponível em: http://esabsp.esab.net/files/Fact\%20 sheets/Welding\%20Automation/XA00167320.pdf.

[22] Santos TFA, Lopez EAT, Fonseca EB, Ramirez AJ. Friction stir welding of duplex and superduplex stainless steels and some aspects of microstructural characterization and mechanical performance. Materials Research. 2016; 19(1): 117-131.

[23] Arora A, Nandan R, Reynolds AP, DebRoy T. Torque, power requirement and stir zone geometry in friction stir welding through modeling and experiments. Scripta Materialia. 2009;60(1):1316. http://dx.doi.org/10.1016/j.scriptamat.2008.08.015.

[24] Yan J, Sutton MA, Reynolds AP. Process-structure-property relationships for nugget and heat affected zone regions of
AA2524-T351 friction stir welds. Science and Technology of Welding and Joining. 2005;10(6):725-736. http://dx.doi. org/10.1179/174329305X68778.

[25] Husain MM, Sarkar R, Pal TK, Prabhu N, Ghosh M. Friction stir welding of steel: heat input, microstructure, and mechanical property co-relation. Journal of Materials Engineering and Performance. 2015;24(9):3673-3683. http://dx.doi.org/10.1007/ s11665-015-1652-5.

[26] Lienert TJ, Stellwag WL Jr, Grimmett BB, Warke RW. Friction stir welding studies on mild steel. Welding Journal-New York. 2003;82(1):1S.

[27] Geremia Redutores. Redutor GD com mancal axial: catálogo de aplicação e especificação. 2016 [Acesso em 30 jun. 2016]. Disponível em: http://www.geremiaredutores.com.br/catalogos. 